\title{
Gradhiva
}

GRADHIV

Revue d'anthropologie et d'histoire des arts

$31 \mid 2020$

L'idéal du musicien et l'âpreté du monde

\section{Combien coûte une chanson? La culture du prix et le prix de la culture}

Carlos Fausto

Traducteur : Clotilde Meyer

\section{OpenEdition}

Journals

Édition électronique

URL : http://journals.openedition.org/gradhiva/5116

DOI : 10.4000/gradhiva.5116

ISSN : 1760-849X

Éditeur

Musée du quai Branly Jacques Chirac

Édition imprimée

Date de publication : 2 septembre 2020

Pagination : 130-142

ISBN : 978-2-35744-131-6

ISSN : 0764-8928

\section{Référence électronique}

Carlos Fausto, « Combien coûte une chanson? La culture du prix et le prix de la culture », Gradhiva [En ligne], 31 | 2020, mis en ligne le 31 mars 2021, consulté le 01 avril 2021. URL : http:// journals.openedition.org/gradhiva/5116; DOI : https://doi.org/10.4000/gradhiva.5116 
Traductions inédites

Combien coûte

une chanson?

La culture

du prix et le prix

de la culture*

*

Ce texte a fait l'objet d'une première

publication en anglais sous le titre

«How Much for a Song? The Culture

of Calculation and the Calculation

of Culture», chapitre 5 du livre

Ownership and Nurture. Studies

in Native Amazonian Property

Relations, dirigé par Marc Brightman

Carlos Fausto et Vanessa Grotti.

New York, Berghahn Books, 2016,

p. 133-155.

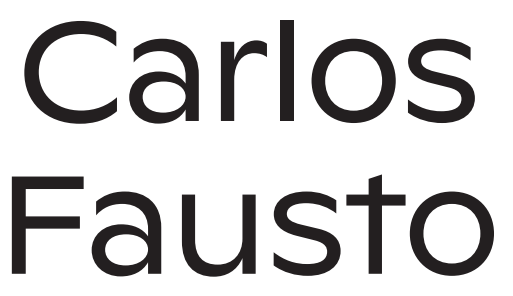


Le texte présenté ici traite d'une question - à qui appartient la culture, et de quelle nature est cette «propriété»? - que Gradhiva a déjà abordée à plusieurs reprises, notamment dans le dossier de son numéro 12 intitulé La Musique n'a pas d'auteur paru en 2010 et coordonné par Christine Guillebaud, Julien Mallet et Victor A. Stoichita.

À partir des données collectées chez les Kuikuro dans le sud de l'Amazonie brésilienne, Carlos Fausto, professeur à l'Université Fédérale de Rio de Janeiro et directeur de recherche au CNPq, dresse dans l'article traduit ici un tableau des formes de commerce pratiquées dans cette aire indigène.

Les Kuikuro - un groupe de langue carib formant l'une des composantes de l'ensemble pluriethnique du Xingu - se distinguent par une pratique intensive de l'échange inter et intra-tribal, toujours assortie de paiement immédiat, soit en biens de prestige tels que des ceintures de perles ou de coquillages, soit en monnaie nationale. Chez eux, tout, ou presque tout, se «vend» et se rétribue. Voilà qui sonne familier. Dans ce régime de valeurs cependant, le prix d'un objet - tangible ou intangible - se calcule en fonction de la valeur des relations qu'il permet de créer ou dont il est l'expression: ici, on désire du lien, non des biens.

Chez les Kuikuro, la maîtrise des chants rituels est source de grand prestige, et leur transmission a un prix. Celle-ci impliquait jadis un contrat bien étrange entre maître et apprenti: chacun devait promettre de «tromper» l'autre. C'était une manière de conjurer par antiphrase le risque d'une interprétation erronée et de contraindre l'apprenti à «rentabiliser» son investissement en le transmettant à son tour. Cette «vente» d'un chant suppose une grande confiance entre partenaires. Pour cette raison, la transmission se fait entre proches parents et l'expertise reste l'apanage de certaines familles.

Mais avec l'avènement des cassettes audio, les jeunes apprennent par eux-mêmes, sans avoir à rémunérer un maître. Cette banalisation entraîne aussitôt une forte dépréciation du répertoire et des maîtres qui le portent. Pour conjurer la disparition progressive des chanteurs de tradition, capables de mémoriser des centaines de pièces à la lettre et à la note près, le chef kuikuro et l'anthropologue Carlos Fausto lancent un projet d'archivage digital du corpus rituel. Cela crée deux difficultés. La première est de trouver un standard de paiement par chant qui soit accepté par tous les maîtres. La seconde est de savoir à qui appartient ce corpus enregistré. À tout le groupe, disait le chef, ce qui voulait dire, à lui-même. E† qui pourrait alors y avoir accès, sinon lui? Les copies seraient confiées au musée de l'Indien à Rio et au centre kuikuro de documentation. Mais en «muséifiant» la tradition on signait sa mort. II fallait donc la rendre accessible, dans les termes de la tradition: à chaque écoute de ces enregistrements, un paiement serait attribué au possesseur de ces chants.

Cette gestion du copyright est le prix que les Kuikuro continuent d'accorder à la relation instaurée entre maître et apprenti comme à la capacité à la créer, un signe que la tradition et le régime de valeur dans lequel elle s'inscrivait résistent à leur dissolution promise dans un marché monétarisé. 
Quand je lui expliquai que, s'il donnait du poisson, il en aurait moins qu'avant, Robtokti me répondit:

Quand un Suya donne quelque chose à quelqu'un, il n'en a pas pour autant moins pour lui. Quand je donne du poisson à mon frère, il me revaut toujours ça. Donc si j'en ai 10 et que je lui en donne 3, il me donnera plus de poisson quand il ira pêcher. Donc ça fait 10+3, et non pas 10-3.

(Ferreira 1997)

Le premier missionnaire à avoir exploré la Nouvelle-Zélande, Samuel Marsden, fit l'hypothèse que le peuple maori descendait d'un groupe de juifs errants: il en voulait pour preuve le fait qu'ils «possèdent un grand penchant naturel pour le commerce; ils achètent et vendent tout ce qu'ils ont» (Hanson 1989: 892). Si Marsden avait débarqué dans le haut Xingu en ce même an de grâce 1819, il en aurait probablement conclu que les Xinǵuano aussi étaient les rejetons de l'une des tribus perdues d'Israël, à en croire leur prédilection similaire pour le "commerce». Gertrude Dole (1956-1958: 125), la première chercheuse à travailler auprès des Kuikuro, l'un des peuples composant le système multiethnique du haut Xingu, a en effet noté dès les années 1950 qu' «ils sont obnubilés par l'échange de biens et de services, ainsi que par l'équilibre des comptes entre eux». Et d'ajouter, un peu plus loin: «Presque tout don ou service rendu exige rétribution. Le partage et le don gratuit n'existent quasiment pas chez les Kuikuro.» (ibid: 126)

Ce tableau d'une société indigène amazonienne obsédée par l'échange de biens et de services et par l'équilibre des comptes est assez peu commun. Lorsque, à mon tour, j'ai commencé mes recherches auprès des Kuikuro, j'ai subi un véritable choc culturel, comme en témoignent ces notes du 23 juillet 2000 :

\section{Il faut toujours «payer»-en général immédiatement, car, dans le cas contraire, ils font des remarques $d u$ genre: "quand est-ce que tu comptes payer?" Aujourd'hui, par exemple, quand Bruna [Franchetto] a offert un cadeau au grand-père [Agatsipá], celui-ci s'est aussitôt excusé pour le défaut de paiement; Bruna a dû insister, lui expliquer que le cadeau était un don gratuit, que c'était comme ça ches les kagaiha [les Blancs]. \\ (Fausto 2000, notes de terrain)}

Le concept de gratuité comme tradition spécifique aux Blanes on appréciera le paradoxe!

Auparavant, j'avais travaillé auprès des Parakanã, un peuple amérindien de langue tupi, qui n'avaient que très peu d'objets, jamais dotés de valeur transcontextuelle. Leur système numérique était limité à quatre entités: un seul, pair, impair et plusieurs. Il n'y avait aucune unité de compte, ni aucune forme rituelle organisant le transfert de biens. Il existait certes un verbe désignant l'échange de choses identiques (-ponekwan), ainsi qu'un terme siǵnifiant «compenser»ou «payer en retour» (-wepy), mais les actions ainsi décrites ne définissaient pas un code de conduite régissant les relations quotidiennes. Quant à moi, chercheur étranger, je n'avais pas constamment à calculer le coût des questions et autres requêtes que j'adressais à mes «informateurs».

Au début de mes recherches auprès des Kuikuro, j'ai donc eu l'impression d'être sur une autre planète, tant j'avais du mal à assimiler une logique dans laquelle la moindre chose avait un coût. Parfois, l'exigence de paiement s'inscrivait dans un domaine ou un type de relation encadré par des prix fixes; d'autres fois, il fallait calculer ce prix au pied levé, avec toujours le risque de donner trop ou pas assez ${ }^{1}$. Presque tous les services et objets peuvent entrer dans le cadre de ce système de «paiement»ainsi que les Kuikuro traduisent généralement le mot ihipügü. Services fournis par des chamanes, des chanteurs, des messagers, mais aussi peinture corporelle, lamentation rituelle, fin de la période de deuil, faveurs sexuelles: tout cela a un «prix». Seuls font exception les aliments, frais ou transformés, qui restent presque toujours en dehors de ce système ${ }^{2}$. D'où un autre paradoxe: si, pour ma part, j'avais l'impression qu'au village «tout avait un coût», les Kuikuro disaient la même chose de la ville, où il fallait payer pour manger et dormir ${ }^{3}$.

Pour mieux comprendre ce monde où «tout a un prix », où rien n'est «gratuit», il convient de décrire les différents domaines et sous-systèmes du régime de valeur. Je donnerai dans ce texte un aperçu très général de ce système, en commençant par les activités rituelles qui supposent une standardisation de la valeur. Puis, je m'intéresserai à une activité «commerciale» ritualisée au cours de laquelle des équivalences sont négociées de façon improvisée, pour traiter ensuite du paiement des services chamaniques, des compensations matrimoniales et de la transmission du savoir. J'évoquerai enfin des situations contemporaines dans lesquelles les critères de valeur sont renégociés pour s'adapter à une logique et des acteurs nouveaux (moi y compris).

\section{LE TEMPS ENTRE DONNER ET REGEVOIR}

Le système du haut Xingu est formé de multiples unités politiques autonomes réunies en un ou plusieurs villages. Ce qui définit leur autonomie politique est la présence d'un chef à leur tête et leur capacité d'organiser un rituel intertribal ou d'être convié à participer à un tel rituel orchestré par une autre unité politique. Ces unités sont caractérisées par des différences linguistiques, que ce soit de lanǵue ou de dialecte, même si l'unité linǵuistique ne recoupe pas nécessairement l'unité politique ${ }^{4}$. La langue sépare mais unit aussi. Des macroblocs formés des locuteurs d'une même languue coïncident en partie avec le monopole exercé sur certaines spécialités artisanales : par exemple, les Caribe produisent des colliers et ceintures en coquilles d'escargot, les Arawak sont potiers tandis que les Kamayura et les Aweti de langue tupi sont, respectivement, spécialisés dans la fabrication d'ares en bois noir et de sel végétal ${ }^{5}$.

Il y a équivalence entre les produits les plus raffinés et prestigieux de chacune de ces industries. Par exemple, un grand pot wauja pour faire cuire la bouillie de manioc équivaut à trois colliers d'escargots caribes (inhu aketühügü); un arc en bois noir vaut une grande ceinture de coquillages (uguka); un petit panier de sel s'échange contre une ou deux ceintures de coquillages, tandis qu'un grand panier vaut un collier. Parmi les objets prestigieux, on trouve aussi la grande coiffe (kahokohugu) avec son diadème de plumes de toucan et de cassique surmonté de plumes d'aigle et d'ara rouge, ou encore les colliers en griffes de jaguar. Autrefois, les lames de hache en granit avaient aussi leur place 
parmi les objets de luxe, mais elles furent rapidement remplacées par les outils en métal introduits lors des expéditions allemandes de la fin du XIX ${ }^{\mathrm{e}}$ siècle. Le sel végétal a également eu tendance à se dévaloriser, sans pour autant disparaître complètement, avec l'introduction dans la région du sel industriel. Parallèlement, les perles de verre en provenance de l'ancienne Tchécoslovaquie sont venues compléter sans réellement concurrencer les colliers et ceintures de coquillages. En effet, l'industrie de la coquille d'escargot profita de l'apparition de nouveaux outils - couteaux, aiguilles, pierres à polir -, même si cette amélioration technique contribua probablement à une certaine dévaluation des objets ainsi fabriqués, comparativement à la poterie, par exemple. Celle-ci, en effet, ne subit que modérément la concurrence d'un nouveau produit de luxe: les casseroles de 100 litres en aluminium ${ }^{6}$.

Ce qui détermine la valeur de ces objets - s'ils sont plus ou moins «chers» (tihipükoinhü) - n'est pas leur utilité ou leur rareté. Ni même le temps de travail abstrait nécessaire à leur production. En tout cas, ce n'est pas en ces termes qu'en parlent les Kuikuro. Ils se réfèrent plus volontiers à l'effort, la souffrance, la difficulté qu'implique leur fabrication - une considération plus proche de la notion juridique de pretium doloris ( de la douleur») que de la théorie de la valeur travail. Ainsi, par exemple, s'ils considèrent que leurs colliers valent cher, c'est parce que leur matériau est difficile à perforer, très difficile à couper et donc susceptible de blesser les mains de l'artisan. De même, les Aweti vendent cher leur sel eu égard à l'effort et au danger qu'implique sa production: non seulement elle nécessite une longue exposition au soleil, mais le risque existe aussi de se faire mordre par un serpent, électrocuter par une anguille électrique ou de se retrouver couvert de sangsues ${ }^{7}$. La cherté de ces objets continue aujourd'hui d'être justifiée de cette façon, alors même que l'introduction des outils en métal, des armes et des vêtements a énormément facilité leur fabrication ${ }^{8}$.

Les coquilles d'escargot et l'arǵile sont toujours les principales matières des objets servant aux paiements rituels entre tribus. Toute fête réunissant plusieurs unités politiques est préparée par une invitation transmise par trois messagers. Si l'invitation est acceptée, ceux-ci incitent le village invité à choisir trois chefs, qui guideront le groupe désigné jusqu'au village hôte. À l'issue de la fête, juste après la fin des combats, chacun de ces chefs dépose des «choses» (engiko) au sol à l'intention des messagers qui les ont invités - ce en respectant l'ordre hiérarchique (le premier chef offre des objets au premier messager, etc. ${ }^{9}$ ). Ce n'est pas là un simple échange de biens : chaque messager, après avoir emporté chez lui ce qu'il a reçu, revient sur la place demander au chef invité: «Qu'es-tu venu chercher? 》 (tü kaema egei enhügü? )

Classiquement, un invité kuikuro apportera des ceintures en coquilles d'escarǵot et, si la fête a lieu dans un village wauja, demandera de la poterie en retour. Cet échange est exempt de tout jugement sur la qualité des objets. Que ceux-ci soient bien ou mal faits, grands ou petits, l'échanǵe est obligatoire et se fait sans marchandage. C'est pourquoi, dit-on, le messager du village hôte, dans le laps de temps séparant nécessairement le moment où il reçoit et celui où il donne en retour, est particulièrement angoissé lorsqu'il repasse chez lui en tentant de deviner ce que le chef des invités va lui demander.

Les biens manufacturés ont été peu à peu introduits dans ce système. Il y a quelques années, une femme matipu, après avoir offert des ceintures d'escargots, a demandé à un messager kuikuro une «vieille bicyclette», c'est-à-dire, dans le langage des demandes rituelles, un vélo en bon état. En 2010, une jeune femme kuikuro qui avait guidé les invités jusqu'à un village
1. Au début de mon travail de terrain, j'ai assisté à la scène suivante: le gendre du maître de la maison où j'étais hébergé arrivait de la FUNAI, où il occupait le poste de responsable de la radio. II avait rapporté avec lui tout un tas de matériel

technologique, technologique, notamment une chaîne hi-fi,
un téléviseur et une antenne parabolique. Ses frères n'étant là pour l'aider à installer I'antenne, il demanda du renfortà un jeune garçon. La mission une fois accomplie, je vis le gendre rentrer dans la maison et se mettre à fouiller fébrilement dans ses affaires, sans savoir ce qu'il cherchait. Je lui demandai ce qu'il se passait et, tout en sortant un bermuda tout neuf de son sac, il m'expliqua qu'il devait payer (ihipügü) le garçon pour son travail. Il venait de s'installer au village à la suite de son mariage et s'efforçait de s'imposer comme expert en produits manufacturés, mais pour finir il paya trop cher une tâche qui ne valait normalement pas plus d'un ou deux savons ou de quelques piles.

2. La nourriture est un élément de rétribution fondamental pour un travail collectif. Cette contre-prestation, appelée endugu, n'est pas considérée comme un paiement. Nourriture et boisson sont offertes par le maître de la maison ou du jardin où le travail est exécuté.

3. Le spectre de la monétisation de la vie du village ne menace en réalité que la sphère alimentaire. Le problème n'est pas

l'introduction de la monnaie dans les circuits «traditionnels» d'échange, mais son interférence avec le partage alimentaire. Un ami kuikuro m'a un jour raconté I'histoire d'un Xinguano qui, de passage dans un village bakairi, se retrouva affamé parce que là-bas, au lieu de partager la nourriture, on la vend sur un petit marché installé en plein territoire indigène. Dans un village kuikuro, le partage quotidien de nourriture entre parents proches et affins est complété par des formes ritualisées permettant aux membres plus éloignés de la communauté de demander de la nourriture, de se faire prêter un jardin ou un coin de rivière équipé de pièges à poissons. Ces procédures sont appelées inko ihatoho («pour montrer les choses»).

4. Par exemple, les Kalapalo se répartissent en deux unités politiques autonomes, correspondant aux villages d'Aiha et de Tanguru.

5. Les Mehinaku produisent aussi du sel végétal. Je suppose qu'ils ont renoncé à leur monopole sur sa production au cours du processus d'intégration des Aweti au système xinguanien. En ce qui concerne les parures en coquilles d'escargot, il y aurait eu jadis une distinction entre les colliers des Kalapalo et les ceintures des Kuikuro.
6. Les casseroles d'aluminium de 70 et 100 litres sont devenues des objets de paiement, mais ne servent guère d'ustensiles pour faire cuire la bouillie de manioc. S'il reçoit une casserole en guise de paiement, I'organisateur d'un rituel doit offrir à manger à la communauté encore une fois avant tout usage personnel de l'objet.

7. Les Kuikuro partagent l'avis des Aweti quant à la valeur de leur sel. D'après Figueiredo (2010: 99), les Aweti expliquent toujours son prix élevé dans les échanges intercommunautaires en arguant des «dangers et de l'épreuve physique que les femmes affrontent au cours du processus de fabrication».

8. Avant, par exemple, la pierre utilisée pour percer les coquilles d'escargot était acquise auprès des Kalapalo, qui se la procuraient eux-mêmes au terme d'un long voyage le long du rio Culuene

jusqu'à un site appelé kugugi, que je situe à 60-70 kilomètres au sud de l'actuelle frontière du parc indigène du Xingu.

9. Ces «choses» ne sont pas désignées comme un «paiement» (ihipügü). Les chefs des visiteurs les appellent uakandokongo («le compagnon de mon tabouret»), car le tabouret est le symbole de leur statut: le chef des invités (hagito anetügü) est celui qui va «sur le tabouret» (tahaguhongo).

L'hôte-messager, de son côté, nomme en général ce paiement uetidzingagü (que l'on pourrait traduire par: «le fait que j'aie été messager »). Les colliers et les ceintures doivent être placés au sol en une ligne verticale reliant le chef des invités et les messagers. 
yawalapiti - connu pour réunir différentes tribus xinguaniennes et concentrer de nombreux biens industriels - a réclamé au messager 150 litres de pétrole en échange des deux ceintures qu'elle-même lui avait données.

\section{LES MARCHÉS DOMESTIQUES}

Le système d'échanges rituel est chapeauté par une sorte d'unité de compte, qui encadre les relations entre unités politiques. Dans le cas des Kuikuro, cette norme de valeur s'exprime principalement en ceintures et colliers d'escargots. De tous les objets en usage chez eux, les parures en coquilles d'escargot, du fait de leur résistance, de leur capacité à se transporter et se dénombrer facilement (on compte le nombre de cordons), constituent le meilleur équivalent à l'idée de monnaie, comme me l'expliqua un Kuikuro à qui j'avais demandé si je pouvais utiliser de l'arǵent pour participer aux échanges rituels appelés luki: «Bien sûr, l'argent, c'est les ceintures de coquillages des Blancs» (tamatehe, kaǵaiha ugukasü hegei tinhegui).

Le luki est une pratique commerciale ritualisée interne à un villaǵe, qui peut aussi prendre la forme d'un rituel public intertribal. Luki signnifie «hirondelle», à l'image du groupe de gens allant, à cette occasion, de maison en maison tel un vol d'hirondelles. Il a lieu plus fréquemment à la saison des pluies, mais peut dans l'absolu se dérouler à n'importe quel moment de l'année, notamment quand un groupe rentre de la ville, chargé de biens manufacturés. Quant au rituel public, il a lieu quand les habitants d'un village se rendent dans un autre village pour commercer: la mécanique de l'échange est ǵrosso modo la même, à ceci près que le marché n'a plus lieu à l'intérieur de la maison mais sur la place centrale ${ }^{10}$.

Le luki, comme pratique domestique ritualisée, consiste en une sorte d'équilibrage des comptes immédiat entre les parties, ce qui évite les attentes démesurées et limite les demandes qu'une personne peut faire à une autre. Tout commence par une banale conversation, au cours de laquelle les interlocuteurs expriment leur désir commun de commercer. Si l'idée est retenue, ils se mettent en quête, afin de présider le groupe, d'une personne qui a déjà «été sur le tabouret» (tahaguho tütegatinhüpe) - c'est-à-dire quelqu'un qui a déjà tenu le rôle de chef des invités lors d'une fête intertribale. Puis ils se rendent chez l'un des chefs du village, qui invite le chef du groupe à s'asseoir sur un tabouret. Un par un, les participants déposent des objets par terre devant lui. L'acte de placer l'objet au sol est décrit par le terme-akanenügü («faire dépôt»), expression également employée pour la vente d'objets artisanaux à la ville. Ensuite, le chef du groupe demande «que veux-tu en échange de ça?» (tü kaema? ) et le vendeur fixe un prix, généralement exprimé par une formule possessive à la première personne: «ma ceinture», «mes chaussures de foot», etc. ${ }^{11}$. À ce stade, les participants doivent décider s'ils prennent l'objet proposé. Si personne ne le fait, le «vendeur»va immédiatement chercher un autre objet et formule un nouveau souhait. Tout cela est censé se dérouler rapidement, sans pause, ce qui incite les habitants à exposer leurs biens les plus précieux, souvent cachés dans des sacs et des valises dans les coins les plus reculés de la maison. Quand le rythme des échanges commence à retomber, le cycle est terminé. Les transactions s'arrêtent. Le groupe se rend alors dans la maison suivante, en se déplaçant autour de la place centrale dans le sens contraire des aiguilles d'une montre, et ainsi de suite jusqu'à atteindre la dernière maison ${ }^{12}$. Enfin, les «hirondelles» de ce marché domestique itinérant se dispersent.
Quasiment n'importe quel objet peut être échangé au cours du luki: des colliers de coquillages et des téléphones portables, des cannes à pêche et de l'huile de péqui, de l'argónt ou même de la nourriture. En revanche, le marchandage est interdit: c'est la personne qui a déposé l'objet au sol qui «fixe le prix».

La seule forme de calibrage consiste à placer quelque chose et demander l'objet souhaité en échange. Si personne n'accepte la transaction, le premier objet est enlevé et remplacé par un autre de valeur supérieure, en redemandant le même objet ${ }^{\mathbf{1 3}}$. Il existe également une certaine normalisation de la valeur exprimée en bandes de colliers de perles tchèques. Au début des années 2000, par exemple, un savon valait quatre bandes, tandis qu'une longueur de tissu s'échangeait contre huit.

Bien que presque tout circule au marché luki, il y a tout de même quelques exceptions. Entre autres éléments exclus, on trouve: les objets chamaniques, les objets rituels et les enregistrements de chants rituels. Si quelqu'un a payé pour enreǵistrer des chansons, il n'a pas le droit de vendre cette connaissance matérialisée sur une cassette audio. S’il veut la transmettre à une tierce personne (moyennant rétribution), il doit le faire de vive voix. Ce savoir doit d'abord s'être ancré dans la mémoire, ou plutôt, comme disent les Kuikuro, «incorporé dans le ventre».

\section{SERVICES CHAMANIQUES}

Le système de valeurs standardisé régissant l'échange rituel se retrouve à des niveaux inférieurs d'inclusion et dans d'autres contextes, rituels ou non ${ }^{14}$. Ainsi, par exemple, dans le cas de la thérapie chamanique visant à guérir l'âme d'un malade, prise en charge par trois à cinq chamanes, la rétribution attendue est bien établie: le chamane principal reçoit normalement un collier ou une ceinture de coquillages de grande qualité, et le chamane qui tient la poupée utilisée pour guérir le malade reçoit un collier de perles à bandes multiples. Les autres assistants reçoivent quelques bandes de perles ou une ceinture en coton ${ }^{15}$. Si l'on ne dispose pas de collier, on improvise: j'ai ainsi vu un homme payer le chamane principal avec un vieux vélo (quand les bicyclettes étaient encore rares au village). Contrairement à la plupart des paiements de biens et de services, ces rétributions ne sont pas nommées ihipügü. Mais plutôt: ihatoho, qui pourrait se traduire par: «fait pour (re)compter ${ }^{16}$ ». Si le paiement n'est pas fait immédiatement à l'issue de la séance chamanique, l'esprit auxiliaire du chamane peut se retourner contre son maître (le chamane) ${ }^{17}$. Le prix des services chamaniques est relativement stable, et tout adulte veille à mettre de côté un petit stock de coquillages ou de parures de perles pour couvrir le coût d'un éventuel traitement. Même les thérapies les plus ordinaires, qui ne sollicitent qu'un seul chamane pour extraire du patient les «flèches de l'esprit», nécessitent parfois le paiement substantiel d'un collier ou d'une ceinture de coquillages ${ }^{18}$. Dans le cas des nouveau-nés, on prend des dispositions avant que la situation se dégrade. Quand le bébé est malade pour la première fois, les parents choisissent un chamanepédiatre (ipagisü) et lui versent en guise de paiement forfaitaire un collier ou une ceinture de coquillages. Ce paiement vaut pour le suivi de l'enfant jusqu'à ce qu'il commence à courir. Durant tout ce temps, on peut solliciter l'intervention du chamane sans avoir à le payer de nouveau. S'ils le souhaitent, les parents peuvent ensuite payer un surplus pour étendre la couverture jusqu'aux 5 ans de l'enfant environ. Comme tout autre paiement fait aux 
chamanes, ces biens sont nommés ihatoho. Ils ont toutefois une particularité : le chamane est tenu de les conserver jusqu'à la fin de la période concernée, et si l'enfant vient à mourir, il doit rendre aux parents l'objet précis qu'il a reçu de leur part - pas un autre de la même valeur ${ }^{19}$.

Tous ces ihatoho sont considérés comme des paiements versés aux chamanes et à leurs «enfants », c'est-à-dire aux esprits qui leur confèrent le pouvoir de soigner et qui leur sont consubstantiels 20 Les paiements destinés à ces personnes doubles obéissent à un schéma régulier, connu de chacun, y compris des esprits. Ces dernières années, cependant, une recrudescence de nouveaux prophètes dans la région a, entre autres choses, remis en question cette échelle de valeurs. Le cas le plus significatif date d'il y a environ cinq ans: un ancien champion de lutte se mit soudain à soigner au nom de Dieu, combinant de façon inédite des éléments traditionnels du chamanisme et des innovations de son cru, inspirées du christianisme ou librement interprétés de la cosmologie xinguanienne. Les tarifs de ses prestations connurent une soudaine inflation: un Kuikuro, malade depuis plus d'un an des suites d'une initiation chamanique ratée, alla jusqu'à lui payer treize colliers de coquillages. S'étant forgé une réputation dans tout le village, il déclara que son esprit - Dieu - n'accepterait plus pour ihatoho que des billets de banque. Il aurait ainsi exigé 2000 reais pour soigner le chef des Kĩnsêdjê (Suyá) - somme qui aurait été payée par le conseil local de Querência, une petite ville située en bordure du parc du Xingu, où les Indiens représentent une grande part de l'électorat.

Ce n'est pas ici le lieu de raconter comment le chamanisme traditionnel a répondu aux innovations de ce prophète, de ce «Maître » autoproclamé21. Notons seulement que l'un des facteurs de sa chute fut précisément la dérégulation du système traditionnel de paiements par l'introduction de monnaie fiduciaire. L'exigence de Dieu d'être payé uniquement en billets de banque blessa un ǵrand nombre de gens qui se trouvèrent exclus de la thérapie, du fait de la difficulté à se procurer les «feuilles» (tagiü) des Blancs, ainsi que les Kuikuro appelaient originellement le papier-monnaie 22. Même si beaucoup de Xinguano ont des comptes en banque, les gens n'épargnnent pas l'argent comme ils le font avec les colliers ou ceintures de coquillages. Ils préfèrent dépenser tout ce qu'ils gagnent, convertir leur arǵent en biens manufacturés qui entrent dans le système d'échange, les reliant ainsi à leurs proches.

Ils ne se soucient guère de la dimension morale attachée à l'argent, mais plutôt des moyens de s'en procurer. L'argent n'est rien d'autre que le collier de coquillages des Blancs, et même si, pour ma part, j'ai toujours été réticent (pour des raisons morales, pour le coup) à payer des services en argent, c'est pourtant précisément ce qu'ils attendaient de moi.

\section{COMPENSATIONS : \\ LE PRIX DE LA MARIÉE}

En plus de servir de monnaie d'échange dans les relations rituelles intertribales et les thérapies chamaniques, les objets de luxe sont utilisés comme compensation matrimoniale. En principe, chez les Kuikuro, le gendre doit gratifier de colliers d'escargots tous ses beaux-parents, y compris les parents classificatoires, pour les dédommager du fait qu'il aura des fils avec sa femme ${ }^{23}$. Le paiement est effectué par la mère du marié dans le cas d'un premier mariage de la fille, notamment si elle sort tout juste de la période de réclusion suivant ses premières rèǵles et s'est fait couper la frange. Quand une fille se marie alors qu'elle a déjà
10. Le luki intertribal est surtout une affaire d'hommes. À leur arrivée, les chefs des invités déposent leurs objets au sol sans dire ce qu'ils veulent en échange. Les chefs des hôtes sont ainsi obligés de les prendre. Ils ne s'accordent sur un «prix» que dans un second temps, en privé. Le lendemain a lieu un autre luki, cette fois-ci tenu par les femmes, mais à l'intérieur des maisons (voir Basso 1973: 135-138)

11. La personne peut aussi tout simplement répondre: «À toi de voir» (eingunkgike), repondre: «A toi de voir» (eingunkg
laissant à qui prendra l'objet le soin de décider ce qu'il donnera en échange.

12. Le circuit suit parfois une autre logique: on commence entre deux maisons, et si quelqu'un entre dans le cercle d'habitations, les participants se rendent d'habitations, les participants se rendent
chez cette nouvelle personne, traçant ainsi des diagonales à travers la place du village.

13. Certaines personnes, qui se sentent obligées d'exposer leurs biens mais souhaitent en fait les garder, demandent exprès des objets très précieux en échange. Ce procédé, toutefois, signale que la personne est trop attachée (tüitunginhü) à ses objets - ce qui n'est évidemment pas un compliment.

14. À la fin d'un rituel, la coutume veut que l'on paye le maître du rituel pour avoir nourri la communauté des gens et des esprits pendant tant d'années. Chez les Kuikuro, chacune des personnes que l'on appelle des «corps» (ihü) - c'est-à-dire ceux qui demandent au maître de se charger de la cérémonie - doit lui offrir une grande casserole, aujourd'hui en aluminium, dans laquelle les autres déposeront à leur tour leurs cadeaux.

15. Ce système connaît des variantes: de préférence, le chamane principal fabrique une poupée-esprit, un autre chamane jette un sort (kehege) pour que la poupée prenne vie, tandis qu'un troisième place la poupée sur sa poitrine afin d'aller chercher l'âme là où les «esprits» l'ont emmenée. Les quatrième et cinquième chamanes sont des assistants qui portent les offrandes destinées aux esprits. Toutefois, il n'est pas rare que le chamane qui fabrique la poupée jette aussi lui-même le sort. Quoi qu'il en soit le paiement dépend aussi du temps que dure l'incantation. Aussi les proches de la personne malade doivent-elles être très attentives à celle-ci afin d'évaluer ce qu'elles doivent payer. En tout cas, tous les chamanes doivent recevoir un paiement, sans quoi leur esprit peut se retourner contre eux.

16. La racine -iha signifie «raconter (une histoire)» et «montrer». Lorsqu'elle est utilisée dans une phrase avec le quantifiant hisundu, elle veut aussi dire «compter». Le terme ihatoho désigne l'index, et peut se gloser ainsi: «fait pour montrer ou pointer». Dans ce texte, je le traduis exceptionnellement par «fait pour (re)compter».

17. Les esprits qui se sont emparés de l'âme du malade reçoivent aussi des objets. Au cours de la thérapie chamanique, divers objets prestigieux, notamment la coiffe de plumes rituelle, sont suspendus au centre de la maison puis emmenés par les chamanes assistants au point d'accès vers la demeure des esprits. Ceux-ci s'emparent alors du «double» (akunga) de ces objets.

18. Quand le chamane est appelé au chevet du malade, chez lui, il est payé et doit ensuite revenir deux ou trois fois par jour jusqu'à la guérison complète du patient - et ce, sans toucher de nouveau paiement. Un patient qui se rend lui-même chez le chamane pour une consultation ponctuelle paie avec des objets plus ordinaires.
19. D'après Mutuá Mehinaku, un Kuikuro titulaire d'un master d'anthropologie, ce collier est l'enfant, c'est pourquoi il ne peut être cédé à personne d'autre. Toutefois, cette équivalence entre le collier et l'enfant disparaît quand celui-ci apprend à courir (pas seulement à marcher).

20. L'une des étapes cruciales de l'initiation du chamane est la transmission d'une substance, le nguto, par l'initiateur à l'initié. Cette substance visqueuse est reliée à (et est véritablement) I'esprit. L'initiateur retire le nguto de ses mains, sa bouche, son torse et ses genoux pour l'appliquer aux mêmes endroits du corps de l'initié. C'est cette substance qui permettra au nouveau chamane de retirer les «flèches de l'esprit» (itseke hügi) du corps d'un malade. Une forme directe de transmission existe également: quand une personne est très malade, l'itseke fait pénétrer le nguto à l'intérieur du patient, qui développe ainsi une relation spéciale avec l'esprit concerné. Au demeurant, pour véritablement exercer comme chamane, cette personne devra suivre une formation et une initiation complètes auprès d'un chamane établi. Outre cette forme de consubstantialisation, les chamanes, comme certains malades chroniques, ont une seconde famille dans le monde des esprits, notamment une épouse et des enfants.

21. Sur ce mouvement prophétique, voir: Cardoso etal. 2012; Fausto et Vienne 2014.

22. En 2012, une réunion pan-xinguanienne s'est déroulée dans la ville de Canarana au sujet de l'opportunité de payer les chamanes via les services de santé de l'État. Certains avancèrent que, en tant qu'agents de santé indigènes, ils devaient percevoir un salaire de l'État et ne pas faire payer leurs patients. Mais d'après ce que m'en a rapporté Takumã Kuikuro, lui-même fils de chamane, cette position n'a pas fait l'unanimité. Lui défendait I'idée qu'un chamane ne devrait pas être payé en argent dès lors que l'argent, comme il me l'a expliqué en portugais, «n'est pas visible, ne s'offre pas à nos yeux».

23. Idéalement, un homme doit payer ses beaux-parents à la naissance d'un fils, car ce fils est appelé à être son «substitut» (itakongo), celui qui le remplacera. Ce remplacement intervient à n'importe quel moment, mais il est surtout remarquable en matière de fonctions rituelles. 
eu une activité sexuelle, le mari n'est redevable qu'à ses vrais parents. Comme un jeune homme me l'a un jour expliqué, «c'est comme chez les Blancs : ce qui est usagé est moins cher».

Le cas paradigmatique est celui des mariages entre «chefs» (anetü), qui exiǵent un arrangement matrimonial préalable à la réclusion de la fille. À l'issue d'un rituel intertribal majeur, un oncle maternel ou un lutteur emmène la fille chez le fiancé, où le hamac du jeune homme est décroché puis apporté chez les parents de la fille, où le jeune homme vivra désormais. Le jour même, la mère du jeune homme vient payer la famille de la fille ${ }^{24}$. Ce paiement est parfois appelé le «prix du vagin» (ig̈ügü ihipügü) (Franchetto 1996: 41), ou encore le «prix de l'éducation» (inkǵukipügü ihipügü) - allusion au fait que ce qui est ainsi défrayé est l'effort conjoint des beaux-parents pour élever la fille. Comme pour le chamane-pédiatre, les biens remis à cette occasion - d'habitude des ceintures de coquillages - doivent être conservés par les beaux-parents jusqu'à la naissance du premier petit-enfant, car si le mariage est dissous, les objets doivent être rendus à l'ex-gendre. Si un enfant est né entretemps, on est dispensé de rendre les objets car, pour reprendre l'expression locale, la fille a déjà été «abîmée» (ihetsüpügüu) par son mari ${ }^{25}$.

Du point de vue de la famille de la mariée, ce paiement vient compenser le «soin» (care) mis à élever la fille: les soucis, les réveils nocturnes, l'amour et la nourriture prodigués - en somme, tout ce que recouvre la notion kuikuro de «soin» (igikasi-). La compensation est ainsi assurée par des objets dont la valeur traduit l'effort et la difficulté que nécessite leur fabrication. L'équivalence entre les gens et les choses n'implique pas un calcul abstrait, un «taux de change» selon lequel une certaine quantité de produits aurait une valeur numérique exprimée en «colliers de coquillages» en tant que monnaie. Ce qui est mis en regard est une qualité humaine inscrite au sein même de l'objet: le paiement est la reconnaissance du lien qui unit la fille à ses parents, fruit du «soin» prodigué toutes ces années, plutôt que la simple annulation de ces liens et leur tranfert au mari. Ainsi le service de la mariée et la résidence uxorilocale, loin d'être annulés par le prix de la mariée, s'y ajoutent.

Du point de vue de la famille du garçon, le transfert des objets de luxe est un prolongement du soin apporté à élever leur fils, car la rétribution des beaux-parents atténue la «honte» (ihüsu) du gendre vivant chez sa famille par alliance ${ }^{26}$. Il en tire une plus grande liberté, notamment celle de retirer provisoirement sa femme de chez ses parents. Aux yeux des parents du marié, le paiement est avant tout censé adoucir les années de service de la mariée et réduire la durée de l'uxorilocalité, bien que cela dépende toujours en dernier ressort de la position hiérarchique respective des familles et des contingences propres aux relations interpersonnelles. Le paiement n'implique pas un transfert immédiat des «droits» exercés sur la femme (qui passeraient des parents au mari), ni l'annulation de la dette contractée envers la bellefamille. Bien plutôt, il ouvre la possibilité de créer un nouveau champ relationnel dans lequel les liens entre époux (qui à leur tour deviendront parents) remplaceront progressivement les liens de dette entre affins.

Il existe d'autres formes de compensation, visant à effacer une dette due à un accident. Il y a quelques années, par exemple, lorsque, en jouant avec le feu, un garçon déclencha un incendie qui ravagea cinq maisons, son père paya en compensation à chacun des propriétaires des ceintures et des colliers de coquillages ${ }^{27}$. On ne prétend pas ici que le montant payé équivaut à ce que le feu a détruit: les ceintures ne remboursent pas les biens perdus dans l'incendie. Comme pour le «prix de la mariée», aucune estimation de la valeur réelle des bien perdus, aucun rééquilibrage des comptes n'est possible dans ce cas. Bien plutôt, le but est de rétablir l'équilibre des relations compromis par une dette énorme causant un profond sentiment de «honte»- non plus celle du jeune marié, mais de la personne qui porte le poids de la dette. Les compensations pour blessures ou homicides involontaires obéissent à la même logique, et visent surtout à atténuer le déséquilibre démesuré soudain créé entre les parties ${ }^{\mathbf{2 8}}$.

\section{TROMPERIE : LA TRANSMISSION DU SAVOIR}

Comme la plupart des services, la transmission formelle du savoir doit aussi être rémunérée. C'est notamment le cas du savoir spécialisé des chamanes et des chanteurs, mais cela vaut également pour des compétences aux contours plus flous comme les incantations et les soins médicaux, qui ne sont pas l'apanage des seuls chamanes. Dans le langage poli des relations formelles, ces paiements pour acquérir un savoir ne sont jamais appelés ihipügü, sauf hors contexte. Par exemple, la bonne façon de demander à un chanteur de vous enseiǵner son savoir est de dire: «Trompe-moi avec cette chose que tu n'as pas bien entendue» (uinkguhike hõhõ ahangahesügüki). Le chanteur acceptera, en s'autodépréciant, d'effectivement tromper son élève avec «le peu que j'ai appris » et ne demande rien en échange, sauf précisément que son apprenti ne cherche pas à son tour à le tromper plus tard, quand la première série de chants aura été apprise et qu'il faudra procéder au premier paiement. L'intervalle de temps entre la demande et le paiement varie considérablement en fonction de l'intensité de l'apprentissage. En théorie, durant cette période, le chanteur ne doit pas duper l'élève, ou en tout cas pas trop, dans l'espoir de recevoir une rétribution conforme aux efforts fournis et au savoir transmis. En effectuant le paiement, l'élève reprendra la formule à son compte, en disant que c'est elle ou lui qui trompe le maître.

Cette «tromperie» (augene) n'est pas seulement un genre d'antiphrase, qui voudrait dire le contraire de ce que le mot signifie. La formule en effet rend explicite une virtualité de la relation. Les attentes des acteurs sont à l'aune d'une possible tromperie: il se peut, d'une part, que le savoir ne soit pas transmis correctement; d'autre part que la rétribution ne corresponde pas à la valeur de ce qui a été transmis. Un facteur atténue l'incertitude de cette relation de possible tromperie réciproque: l'existence d'une norme de valeur acceptée de tous et exprimée, une fois encore, en colliers et ceintures d'escargots (ou autres biens équivalents). Cela étant, chacun est différent, et certains sont plus à même de «tromper» que d'autres; en outre, tous les savoirs transmis n'ont pas la même valeur - certains chants ont plus de valeur que d'autres.

Les Kuikuro possèdent quinze ensembles de chants rituels divisés en suites musicales, elles-mêmes constituées d'entre dix à cent chants différents (Fausto et al. 2011). La valeur d'une suite ne dépend pas du nombre de chants qu'elle contient, mais d'une qualité particulière: le fait d'être plus que de la simple musique. Par définition, les chants rituels sont une manifestation des «esprits» (itseke), mais certains sont considérés comme étant eux-mêmes des «esprits» et/ou associés à des temps forts de certains rituels, accompaǵnant des actes qui présentent un risque dans la relation avec les esprits ${ }^{29}$. Ces chants-là sont dits tainpane, terme que les Kuikuro traduisent généralement par «sacré», bien qu'en fait, il soit plus proche du concept anthropologique de tabou. En général, seulement certains des chants d'une suite sont tainpane; mais certaines suites sont entièrement «sacrées». Ainsi, par exemple, parmi les dix-huit suites pour flûtes kağutu - instruments que les femmes n'ont pas le droit de voir - l'une, 
qui ne comprend que dix chants, est considérée comme particulièrement précieuse et chantée à des moments très précis. Elle suscite alors des tensions considérables, car la moindre erreur du chanteur peut lui valoir la mort, à lui ou à un membre de sa famille.

En somme, la standardisation de la valeur ne garantit pas, comme le disait Gertrude Dole, «l'équilibre des comptes entre eux». Le cadrage principal permettant de garantir la convergence des attentes, ce sont les liens de parenté qui assurent la continuité des relations entre maître et apprenti. C'est pourquoi l'on tend à voir se former des familles de chanteurs, au sein desquelles se transmet la connaissance musicale, notamment de parents à enfants. Au demeurant, même dans ces cas-là, l'enseiǵnement doit être rémunéré - bien que de manière moins stricte -, sans quoi, dit-on, l'étudiant ne sera pas capable d'écouter et de mémoriser correctement les chants. Quand on dépense, on pense et se concentre.

L'avènement du maǵnétophone à cassettes il y a quelques décennies a introduit un nouveau mode de transmission : l'on peut désormais, sans entrer en lien avec lui, enregistrer un chanteur résidant dans un autre village, à qui l'on remet immédiatement les biens promis au préalable. On évite ainsi que le processus s'éternise et tout risque d'être «trompé». Les chanteurs disent souvent qu'ils n'enseiǵnent plus aux jeunes aujourd'hui parce que ces derniers ne le leur demandent pas; les jeunes, de leur côté, se disent embarrassés et effrayés à l'idée de s'engager auprès d'un maître et de s'enliser dans un cycle interminable d'apprentissage et de dettes à payer qu'ils ne sont pas sûrs de pouvoir honorer. Le magnétophone leur permet d' «équilibrer les comptes» tout de suite, en reportant sur la cassette la longue implication auprès d'un maître et le poids de ses attentes.

\section{FIXER}

\section{LE PRIX DES CHANTS}

Être un grand chanteur est source de prestige dans le haut Xingu. Les chanteurs en effet jouent un rôle capital dans tous les rituels. Quand ils commencent à être sollicités pour exécuter des chants «sacrés» dans le village hôte lors de rituels intertribaux, ils deviennent célèbres. Chez les Xinguano, la renommée est un enjeu fondamental; elle est conçue, ainsi que Munn l'a montré pour les Gawa, comme une «expansion spatiotemporelle de l'individu» (1986: 117). Lorsqu'un nom circule d'un village à l'autre, celui qui le porte devient tikaginhü, «celui dont on parle beaucoup». Une personne célèbre exerce une grande influence sur l'esprit des autres, car son nom se propage dans le temps et dans l'espace. Les noms se transmettent toutes les deux générations, et un nom éminent - porté jadis par un grand chef, qui laissa de bons souvenirs dans les mémoires - est le meilleur atout possible pour un chef vivant.

Chez les Kuikuro, les chanteurs sont dits eginhoto, de egi («chant») et oto, qui peut se traduire par «maître»ou «détenteur». Comme entume en trio (Brightman et Grotti 2016) ou kandê en Kĩsêdjê (Stockler Coelho de Souza 2016), oto est une catégorie très productive usitée dans de multiples contextes, allant de la relation d'un peuple à son territoire, le lien d'une personne à ses possessions matérielles ou immatérielles (on peut être le «maître» d'un maǵnétophone comme d'un rituel spécifique) ${ }^{30}$. Quiconque possède une spécialité est en général désigné par un terme suivi du vocable oto, sauf le chamane, qui est nommé hüati ${ }^{31}$. Ainsi, l'herboriste s'appelle embuta oto («maître des médicaments»),
24. Le paiement peut être versé à divers «beaux-parents», y compris toutes les personnes que les parents de la fiancée considèrent comme leurs «frères et sœurs» (parmi lesquels des cousins parallèles et croisés). La relation entre beau-paren et beau-fils/belle fille est une relation de strict évitement et, dans les cas où la distance permet plus ou moins de choisir, le beau-parent peut préférer ne pas être «respecté» (itsankgijü) par son jeune gendre ou sa belle-fille. À l'approche d'un mariage, les parents du ou de la fiancé(e) vont rendre les parents du ou de la fiancé(e) vont rendre
visite à leur nouvelle famille avec du bois de chauffage, et leur demandent s'ils souhaitent être respectés par leur beau-fils/belle-fille. Durant les premiers mois du mariage, le jeune couple doit apprendre le nom de tous les membres de leur belle-famille respective, qu'il n'a pas le droit de prononcer.

25. Le verbe s'emploie généralement dans un contexte matériel, comme quand quelqu'un abîme (ihetselü) un objet particulier. La forme intransitive (epetse/ü), on l'emploie pour dire, par exemple, «l'appareil photo a cassé». Si la femme meurt en couches, les biens ne sont pas non plus restitués.

26. La honte est une catégorie fondamentale dans le haut Xingu. Elle est la principale notion/émotion servant à décrire

le comportement moral. Chez les Kalapalo, voir: Basso 1973; Guerreiro 2012. Chez les Mehinaku, voir: Gregor 1977: 219-223. Chez les Wauja, voir: Ball 2007.

27. Le grand-père de l'enfant étant chamane, le père put se tourner vers lui afin de faire des paiements rapides. Ensuite, petit à petit, il fabriqua de nouveaux colliers pour rembourser son emprunt.

28. Il y a de nombreuses années, la harpie féroce d'un chef kuikuro avait tué un enfant, Le chef avait «payé» la famille à titre de compensation pour leur douleur. II s'agit là de sortir d'une situation marquée par des sentiments de honte (ihüsu) et de souffrance (egitamine). La compensation est une façon de reconnaître cette souffrance et non pas d'assumer une responsabilité objective. Il y a quelques années, une femme qui était allée participer à un spectacle dans un centre de loisirs à São Paulo s'est fait écraser par une voiture et a fini paralysée. Les parents réclamèrent un «paiement» aux propriétaires du centre de loisirs, qu aux propriétaires du centre de loisirs, qui
répliquèrent qu'ils n'étaient pas responsables de l'accident. La femme était venue à São Paulo de sa propre initiative et était sortie se balader en ville également de sa propre initiative. Pour ne rien arranger, le chauffeur n'était pas non plus responsable de l'accident. Ce pourquoi, d'après le centre, il n'y avait aucune raison de dédommager les parents. Mais pour ces derniers, le problème était très différent, et n'avait rien à voir avec la responsabilité objective. C'était pour eux une question de honte, et de reconnaissance de la souffrance de la famille.

29. La plupart du temps, ce sont des chants obligeant le «maître» du rituel à fournir un certain type de nourriture, destiné uniquement aux anciens et aux esprits. qui s'était approché trop près de sa cage.
30. Le terme «Kuikuro» vient du nom Ils furent alors appelés Kuhi ikugu otomo, «les propriétaires du lac au poisson kuhi».

31. Le terme ne s'applique pas à la relation d'un chamane avec ses esprits auxiliaires, bien qu'il se réfère volontiers à eux via une forme génitive: «mon esprit est celui du boa» (konto uitsekegüi), pourrait dire un chamane. du lac près duquel ils vivaient au xIx siècle. 
le champion de lutte est un kindoto ( maître de la lutte»), le conteur un akinha oto ( "maître des histoires»), tandis que le chanteur est un eginhoto («maître des chants»). Dans tous les cas cités, il est plus approprié de traduire par «maître»que, par exemple, par «propriétaire», car le terme renvoie à la maîtrise d'un certain savoir plutôt qu'à la possession d'un objet. En tout cas, le savoir comme la possession sont tous deux transmissibles moyennant rétribution.

Comme je l'ai montré plus haut, le savoir musical se transmet toujours en échange de produits de luxe, comme les parures de coquillages ou les biens manufacturés jugés précieux. Par exemple, la grande chanteuse Kanu, qui apprit de sa mère la plupart des chants Jamugikumalu, rétribua d'une casserole en aluminium de 100 litres le chanteur Aǵaku afin «d'avoir» (ngípi) une suite très prestiǵieuse connue sous le nom de Auga imitoho, que sa mère ne connaissait pas. Quand on acquiert un élément de savoir musical - quand on l'a «dans son ventre»-, que l'on est capable de le produire en public au cours d'un rituel, on acquiert le droit de le transmettre à quelqu'un d'autre. On devient alors un eginhoto, indépendant de la source originale du savoir. Dès lors, quand un nouvel apprenti vous paie, vous ne devez rien au maître qui vous a enseiǵné votre savoir en premier lieu ${ }^{32}$.

Ces dernières décennies, les façons de se faire un nom se sont multipliées et écartées du domaine rituel traditionnel. Devenir professeur, aide-soignant, cinéaste, étudiant à l'université, apprendre le portugais, manipuler de l'argent: autant de moyens qui sont aujourd'hui préférés aux anciennes voies menant à la gloire. Les noms circulent aujourd'hui sur Internet; les jeunes ont des profils Facebook, et ils voyagent beaucoup. Au début de mes recherches, il y a plus de dix ans, le chef Afukaká craignnait que le savoir musical des Kuikuro se perde dès lors que, selon lui, les jeunes ne voulaient plus apprendre les chants. J'ai raconté par ailleurs (Fausto 2011) comment ce sentiment d'une "perte culturelle » nous avait conduits à élaborer un projet de documentation consacré spécifiquement à l'univers des chants - projet dont l'ampleur et les particularités constituent un immense défi en matière de mémoire individuelle et collective. Je ne m'attarderai pas sur ce point: ce qui nous intéresse ici, ce sont les dilemmes qui ont surgi après que nous avons décidé de payer les chanteurs pour enregistrer leurs connaissances. Il était entendu que ce paiement serait pris en charǵe par l'Association indigène, créée en 2002 précisément pour gérer le projet, et que les enregistrements seraient réalisés par les vidéastes indigènes formés dans nos cours.

Quatre ans se sont écoulés entre la conception du projet assortie de la création de l'association et les premiers enregistrements. Pendant tout ce temps, deux questions restaient en suspens. D'abord, quel est le prix d'un chant? Ensuite, à qui appartiennent les chants une fois enregistrés - c'est-à-dire, qui a le droit de les apprendre et de les transmettre à son tour. J'aurais pu suggérer que les chants me reviennent, ce qui aurait réglé le second problème, mais n'aurait pas manqué, quant à la première question, de déclencher une surenchère inflationniste risquant de compromettre la mise en œuvre du projet.

Selon le chef Afukaká, les enregistrements appartiennent 《à tout le monde»: ce sont «nos choses » (kukengüko), pour reprendre ses termes. Mais, de l'avis général, rien ici n'appartient vraiment à la communauté (comunidade) par opposition aux particuliers (particular) - deux concepts qui, en portugais, se retrouvent aujourd'hui dans le discours kuikuro. Aussi, tout ce que le chef considère comme un bien commun (tatutolo engü) est en général considéré par les autres comme le bien du chef. Ce qui présente à la fois des avantages et des inconvénients.
D'un côté, le chef est lui-même la forme singulière à travers laquelle le collectif s'incarne, et son absence est ressentie comme un manque. C'est pourquoi le chef est qualifié de tühüninhü, «ce que l'on ne saurait abandonner». Mais de l'autre, le chef, qui possède deux corps, se voit sans cesse accuser d'aǵir en «personne privée» ou plutôt de ne penser qu'à sa famille. Aussi, alors que les ans passaient sans que notre problème soit résolu, le chef Afukaká m'avoua que son peuple ne lui faisait pas confiance ${ }^{33}$.

Un jour, j'ai demandé naïvement à un ǵrand chanteur combien il prendrait pour enregistrer tout son répertoire. Il ne savait pas, me répondit-il, il allait demander à son fils. Un peu plus tard, le fils s'est armé d'un papier et d'un crayon puis a tendu un devis à son père: il avait estimé les chants à 40000 reais. Iǵnorant tout ou presque de la structure et de la tarification de ce savoir, je suis resté perplexe et ai rapidement changé de sujet. Aujourd'hui, je regrette de ne pas avoir essayé de comprendre comment il était parvenu à un tel chiffre, qui au demeurant ne me semble plus si absurde eu égard à l'étendue du savoir de son père. Quoi qu'il en soit, à la suite de ce malentendu, j'ai décidé d'approfondir le sujet afin de définir un critère de valeur. Je me suis mis à étudier la structure interne des ensembles de chants, leur division en suites et leur composition précise, ainsi que les pratiques rituelles et les récits qui leur sont associés. J'avais pour intention d'établir une hiérarchie parmi les ensembles rituels et de convertir les paiements traditionnels en valeurs monétaires. Mais nous avons eu beau multiplier réunions et discussions, nous n'avons fait que tourner en rond. La question restait entière: quel est le juste prix d'un chant?

Finalement, j'ai fait deux propositions. D'abord, une heure d'enregistrement de chants «simples» vaudrait 100 reais et une heure de chants «sacrés» 200 reais. Ensuite, nous enregistrerions tout mais ne laisserions pas les enregistrements à la disposition de n'importe qui. Nous en déposerions une copie aux archives du musée de l'Indien de Rio de Janeiro, et une autre dans un centre de documentation que nous construirions au village ${ }^{34}$. Nous nous sommes mis d'accord sur ces termes. Et le projet a enfin démarré grâce à la nouvelle norme de valeur qui, une fois instituée et appliquée, s'est si bien ancrée dans les mœurs qu'elle a bientôt servi de cadre de base à toute négociation en matière de chants. En 2010 nous suivions toujours le même système de paiement et les chanteurs venaient nous voir d'eux-mêmes, parfois depuis d'autres villages ou même en provenance d'autres peuples de la région ${ }^{35}$. Le chef pouvait enfin dire: «Ils me font confiance.»

Mais l'histoire ne s'arrête pas là. En effet, pour ce qui était de la seconde question, nous n'avions fait que cacher la poussière sous le tapis: à qui appartenaient les chants enregistrés? Les vouer au silence d'un archivage muséologique aurait contredit le sens même du projet, qui était de redynamiser le processus de transmission des chants. Après l'inauguration du centre de documentation en 2007, des jeunes ont insisté pour que les enregistrements soient consultables par tous, mais les chanteurs refusaient de les laisser ainsi à la disposition du premier venu. Que l'Association indigène ait payé ces chants signifiait-il qu'elle les avait désormais dans son ventre? Avait-elle par la même occasion acquis le droit de les transmettre à des tiers? Comme on dirait aujourd'hui, qui détenait le copyright? Et quelle était sa valeur ${ }^{36}$ ? 


\section{CONCLUSION}

Nous avons proposé dans ce texte une description ethnographique d'un village amazonien dans lequel une logique de «paiement» imprègne la société dans de nombreux domaines. Un tel tableau va à l'encontre de l'image traditionnelle des sociétés amazoniennes, souvent dépeintes comme non marchandes, évoluant dans un univers dominé par des pratiques de partage et de don. À mon avis, il ne s'agiit pas là simplement d'un nouvel exemple de la porosité de la frontière entre dons et marchandises. Je préfère plutôt considérer que ce cas illustre la manière amérindienne d'élargiir le champ social par la mise en circulation d'objets (matériels et immatériels) plutôt que par celle de «parties de personnes». Dans un travail antérieur, j'ai développé, pour théoriser ce dernier cas, la notion de «prédation familiarisante»- processus à travers lequel des subjectivités étrangè̀res (trophées, chants, esprits, par exemple) sont violemment capturées à l'extérieur pour être transformées en fils adoptifs à l'intérieur, telles de jeunes proies sauvagees familiarisées, engendrant ainsi une relation de «maîtrise», au sens où l'entendent les peuples amazoniens. Aujourd'hui, je m'efforce de théoriser un réǵime centré sur les objets, variante du modèle centré sur la prédation.

La constitution de la constellation xinguanienne a nécessité de délaisser la prédation au profit d'une sorte de «tissage relationnel», c'est-à-dire la production de relations toujours plus cordiales, par des visites, des dons, des mariages qui construisent progressivement un réseau de similarités plus important que la somme des différences, sans pour autant effacer toutes les différences signnificatives, qui perdurent dans la langue comme dans certaines spécialités artisanales (Fausto et al. 2008). À cet égard, les objets jouent un rôle clé dans la médiation entre peuples d'origines ethnique et linguistique différentes. Le cas décrit ici n'est pas un cas isolé en Amazonie. Sur le plan ethnoǵraphique, le système du haut Rio Negro, composé de populations de lanǵues arawak, tukano et nadehup, en fournit une autre belle illustration (HughJones 2013). Sur le plan historique, le meilleur exemple en matière de standardisation de la valeur est celui du système des Arawak et Pano du piémont andin, où, à l'époque coloniale, des pains de sel servaient de sorte d'équivalent général dans une vaste zone de la forêt (Renard-Casevitz 1993).

La conversion des relations en choses auxquelles on attribue une valeur est un processus bien différent de la conversion d'objets en sujets - un thème plus fréquent des études amazoniennes. Bien entendu, un certain nombre d'objets dans le haut Xingu relèvent aussi de ce dernier cas : on y trouve notamment deux effigies anthropomorphes, ainsi qu'un grand nombre de masques, d'instruments de musique et autre matériel rituel (voir: Barcelos Neto 2008; Fausto et Penoni 2014). Mais ici, ce qui m'a intéressé dans les objets est leur capacité à mettre en relation les gens lorsqu'ils sont transférés et non plus leur éventuelle «substance spirituelle» (soul-stuff), pour reprendre l'expression de SantosGranero (2009). Ces transferts nécessitent un genre de calcul et l'instauration d'équivalences qui peuvent être interprétées comme un système monétaire rudimentaire, ainsi que l'a suggéré Gertrude Dole.

Néanmoins, j'ai voulu montrer ici que ces équivalences ne découlent pas de l'estimation d'une quantité abstraite mais du type de relation impliquée. À cet égard, précisons que le système numérique des Kuikuro (tout comme leur pensée spatiale et géométrique) est assez sophistiqué et leur permettrait tout à fait de calculer des quantités abstraites. C'est pourquoi j'ai d'abord cru que ma propre connaissance arithmétique me serait utile pour calculer la valeur d'un ensemble de chants. Mais ce ne fut
32. II n'y a dans ce cas aucun problème de droit d'auteur, contrairement au cas décrit par Cesarino (2016). Il existe certes des rituels au cours desquels sont composés des chants de dérision, mais ils ne sont pas considérés comme relevant d'un savoir traditionnel et ne sont pas transmis moyennant paiement.

33. Le verbe transitif ikeni- signifie "croire», mais dans ce contexte il peut aussi vouloir dire «faire confiance». On peut dire, par exemple, tikenitümbüngü ekisei anetü, inhalü anetü ikeninümi ihekeni, «ce chef n'est pas une personne digne de confiance; ils ne font pas confiance au chef $)$.

34. Nous rémunérions aussi à la journée tous les services associés au projet: cameraman, transcripteur, traducteur, chauffeurs, etc.

35. Un moment important dans ce processus intervint en 2006, quand le beau-frère du chef Afukaká, lui-même un important dignitaire du village, décida d'enregistrer l'intégralité de son répertoire pour flûtes kagutu. À partir de là, le projet n'inspira plus que de la confiance.

36. De mon point de vue, il restait encore une question: les enregistrements éludaient la pragmatique de la transmission, entraînant une perte du contenu essentiel à

une perte du contenu essentiel à
l'actualisation des chants en situation rituelle. Les enregistrements que les Kuikuro avaient déjà faits sur de vieilles cassettes ne posaient pas tout à fait le même problème, parce que les détenteurs des enregistrements étaient des adultes bons connaisseurs des pratiques rituelles. Mais les jeunes générations, elles, risquaient d'acquérir une connaissance exhaustive de l'univers musical des Kuikuros sans pour autant être capables d'actualiser ce savoir au cours de rituels concrets. La solution que nous avons envisagée fut la suivante: mettre les suites musicales à disposition de personnes précises, en plaçant leur apprentissage sous le contrôle du chanteur dont la voix avait été enregistrée. L'apprenti devrait collaborer avec le chanteur pendant toute la durée de l'apprentissage, et quand il aurait mémorisé une suite complète,

I'Association indigène verserait un nouveau paiement au chanteur. De la sorte, nous retrouverions la boucle des tromperies: nous demanderions aux chanteurs de nous tromper, acceptant le risque ou bien d'être dupes, ou bien d'être nous-mêmes trompeurs, sachant qu'à la fin, nous ne saurions toujours pas la vraie valeur d'un chant. 
pas le cas, car mon projet reposait sur un type de relation inédit - non pas en raison de la technologie d'enregistrement (qui n'était pas nouvelle pour eux), mais parce que, dans ce dispositif, il n'y avait pas d'apprenant: le maître des chants délivrait son savoir pour personne et tout le monde à la fois. C'est pourquoi j'ai finalement eu l'idée de fixer la valeur de cette relation. Une fois établie, cette valeur s'est trouvée faire partie intégrante de son propre contexte de mise en œuvre, et personne ne songea à marchander. Elle devint le coût de notre relation, ou, comme disent les Kuikuro, de la relation «au Projet». Ce tarif n'a pas été fixé en fonction du temps de travail ni de la rareté de l'objet produit, mais en posant une équivalence entre une bande enregistrée et une certaine quantité de monnaie. Les enregistrements, bien entendu, ont une dimension temporelle, mais celle-ci n'est pas entrée en compte dans le calcul. Nous n'avons retenu que le nombre de cassettes mini-DV.

Notre dispositif a eu pour effet de séparer l'enregistrement et l'apprentissage des chants en deux relations différentes. Il existe à présent un détenteur des enregistrements (le Projet, représenté par moi-même, l'association ou le chef du village), mais pas de nouveau maître des chants. Ce qui explique pourquoi les chanteurs exiǵent désormais d'être payés de nouveau quand des gens sont autorisés à apprendre les chants à partir des bandes-son. Il ne faut pas voir là une ruse, mais la prise en compte d'une nouvelle forme de relation à l'âge de la reproduction numérique. Ce qu'ils calculent n'est pas un droit de copie, mais leur capacité à transmettre leur savoir à quelqu'un, à faire que celui-ci ait à son tour «les chants dans le ventre», ce qui ne peut résulter que d'une relation entre des personnes. 
Remerciements:

Ce texte a fait l'objet d'une première version pour le colloque «New Perspectives in Economic Ethnography: Modalities of Exchange and Economic Calculation»», qui s'est déroulé au musée national de Rio de Janeiro en 2011. Je remercie Federico Neiburg et Fernando Rabossi pour leur aimable invitation. Une version ultérieure a fait l'objet d'une présentation à la London School of Economics en 2012 sur invitation de Hans Steinmuller, que je remercie également. David Rodgers en a assuré la traduction en anglais. Cette dernière version, fruit de vingt mois de travail de terrain dans le village d'Ipatse, dans le parc indigène du Xingu, n'aurait pu voir le jour sans un dialogue permanent avec Takumã Kuikuro et Mutuá Mehinaku, à Rio de Janeiro et via Internet. Je remercie la FAPERJ et le CNPq pour le financement d'une grande partie des recherches. Je remercie également pour leur soutien Petrobrás Cultural, le PDPI (ministère de I'Environnement) et I'IPHAN (ministère de la Culture). Enfin, un grand merci à Bruna Franchetto pour m'avoir la première fait rencontrer les Kuikuro (et, encore aujourd'hui, pour son aide linguistique); merci aux Kuikuro de m'avoir accueilli tant d'années parmi eux. 


\section{Bibliographie}

Ball, Christopher Gordon

2007 «Out of the Park Trajectories of Wauja (Xingu Arawak) Language and Culture». Thèse de doctorat, Chicago, University of Chicago.

\section{Barcelos Neto, Aristóteles}

2008 Apapaatai: Rituais de Máscaras no Alto Xingu. São Paulo, Edusp-Fapesp.

\section{Basso, Ellen B.}

1973 The Kalapalo Indians of Central Brazil. New York, Holt, Rinehart \& Winston.

\section{Brightman, Marc et Grotti, Vanessa}

2016 «First Contacts, Slavery and Kinship in North-Eastern Amazonia », in Mare Briǵhtman, Carlos Fausto et Vanessa Grotti (dir.), Ownership and Nurture: Studies in Native Amazonian Property Relations. New York, Berghhahn : 63-80.

Cardoso, Marina Denise, Guerreiro, Júnior Antonio R. et Pereira Novo, Marina

2012 «As Flechas de Maria: Xamanismo,Poder Político e Feitiçaria no Alto Xinǵu», Tellus 23: 11-33.

\section{Dole, Gertrude}

1956-1958 «Ownership and Exchange amonǵ the Kuikuru Indians of Mato Grosso », Revista do Museu Paulista N.S. $10: 125-33$

\section{Fausto, Carlos}

2011 «Mil Años de Transformación: La Cultura de la Tradición entre los Kuikuro del Alto Xingú », in Jean-Pierre Chaumeil, Oscar Espinosa et Manuel Cornejo (dir.) Por Donde Hay Soplo: Estudios Amazónicos en los Países Andinos. Lima: IFEA-CAAPPUCP : 185-216.

\section{Fausto, Carlos,}

Franchetto, Bruna et Heckenberger, Michael 2008 «Ritual, Language and Historical Reconstruction Towards a Linguistic Ethnographical and Árchaeological Account of Upper Xingu Society », in Arienne Dwyer, David K. Harrison and David S. Rood (dir.) Lessons from Documented Endangered Languages. Amsterdam, John Benjamins : 129-158.

\section{Fausto, Carlos, Franchetto,} Bruna et Montagnani, Tommaso

2011 «Les formes de la mémoire art verbal et musique chez les Kuikuro du haut Xingu (Brésil)», L’Homme 197: 41-69.

\section{Fausto, Carlos} et Penoni, Isabel

2014 «L'effigie, le cousin et

le mort : un essai sur le rituel du Javari (haut Xingu, Brésil)», Cahiers d'anthropologie sociale 10: 14-37.

\section{Fausto, Carlos et} Vienne (de), Emmanuel

2014 "Acting Translation: Ritual and Prophetism in 21st Century Indigenous Amazonia», Hau. Journal of Ethnographic Theory 4 (2) : 161-191.

Ferreira, Mariana Kawall Leal 1997 «When $1+1 \neq 2$ : Making Mathematics in Central Brazil », American Ethnologist 24 (1): 132-147.

Figueiredo, Marina Vanzolini 2010 «A Flecha do Ciúme: O Parentesco e seu Avesso Segundo os Aweti do Alto Xingu ». Thèse de doctorat, Rio de Janeiro, Museu Nacional/Universidade Federal.

\section{Franchetto, Bruna}

1996 «Mulheres Entre Os Kuikúro », Revista de Estudos Feministas 1 (1): 35-54.

\section{Gregor, Thomas}

1977 Mehinaku: The Drama of Daily Life in a Brazilian Indian Village. Chicaǵo,

University of Chicago Press.

\section{Guerreiro, Júnior Antonio R.}

2012 «Ancestrais e suas Sombras: Uma Etnoǵraf a da Chefi a Kalapalo e seu Ritual Mortuário ». Thèse de doctorat, São Paulo, Universidade de São Paulo.

\section{Hanson, Allan}

1989 «The Making of the Maori: Culture Invention and Its Logic" , American Anthropologist 91 (4): 890-902.

Hugh-Jones, Stephen 2013 «Bride-Service and the Absent Gift », Journal of the Royal Anthropological Institute 19 (2) : 356-377.

\section{Munn, Nancy D.}

1986 The Fame of Gawa: A Symbolic Study of Value Transformation in a Massim (Papua New Guinea) Society. Cambridge, Cambridge University Press.

\section{Niemeyer Cesarino}

\section{(de), Pedro}

2016 «Doubles and Owners Relations of Knowledge, Property and Autorship among the Marubo », in Mare Brightman, Carlos Fausto et Vanessa Grotti (dir.), Owenership and Nurture: Studies in Native Amazonian Property Relations. New York, Berghahn : 186-208.
Renard-Casevitz, France-Marie

1993 «Guerriers du sel, sauniers de la paix », L'Homme 33 (2-4) : 25-44

Santos-Granero, Fernando

2009 The Occult Life of Things: Native Amazonian Theories of Materiality and Personhood. Tucson, University of Arizona Press.

\section{Stockler Coelho} de Souza, Marcela

2016 «The Forgotten

Pattern and the Stolen Design: Contract, Exchange and Creativity among the Kĩsêdjê », in Mare Brightman, Carlos Fausto et Vanessa Grotti (dir.) Ownership and Nurture:

Studies in Native Amazonian Property Relations. New York, Berǵhahn : 156-185. 